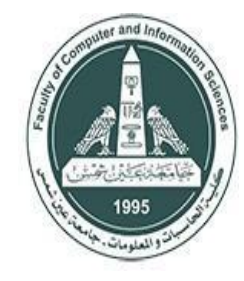

International Journal of Intelligent Computing and Information Sciences

https://ijicis.journals.ekb.eg/

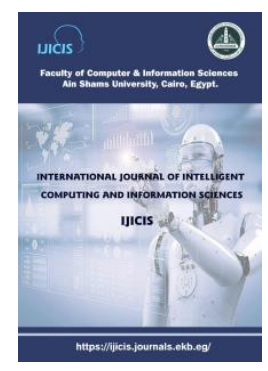

\title{
Integrating Hexagonal Image Processing with Evidential Probabilistic Supervised Classification Technique to Improve Image Retrieval Systems
}

\author{
A.E. Amin* \\ Department of Computer Science, Mansoura University, Mansoura 35516, Egypt \\ Ahmedel_sayed@mans.edu.eg
}

Received 2021-7-4; Revised 2021-9-8; Accepted 2021-9-12

\begin{abstract}
This paper presents a suggested approach to treat a major issue in images classification namely uncertainty. Uncertainty in image classification means some pixels within each cluster are more or less likely to actually belong to this cluster. So, techniques have been used in this paper to deal with the pixels that do not belong to specific regions, helping to raise image retrieval performance. This was done by merging one of the artificial intelligence techniques, which is image processing, with one of the statistical techniques for probability, which is evidential probabilistic. In such contexts, it may be advantageous to resort to two branches: hexagonal image processing based on partial down-sampling of the image resolution in both directions by half using weighted average performance then shifting the remaining pixels in alternate rows. The other is an evidential theory which is rich and flexible formalisms for representing and manipulating uncertain information. Both hexagonal image processing and evidential theory are used to obtain high accuracy in images classification. The hierarchical nature of the hexagonal image processing addressing scheme is exploited to extract features from the image efficiently.

Keywords: Image Retrieval, Evidential Theory, Hexagonal Image Processing, Gabor filters, Evidential C-means.

\section{Introduction:}

Image retrieval content based research is focusing on image classification where classification results are the basis for many digital images processing application. Which called for scientists and practitioners have made great efforts in developing advanced classification approaches and techniques for improving classification accuracy. In general, the beginning of

* Corresponding author: A.E. Amin Department of Computer Science, Mansoura University, Mansoura 35516, Egypt E-mail address: Ahmedel_sayed@mans.edu.eg
\end{abstract}


digital image processing for the purpose of improving transmitted images dates back to the early 1960s.

This paper presents a novel method for regions detect by hexagonal image processing (HIP). Hexagonal lattice is used for image sampling which provides several advantages [1, 2] for example but not limited, the hexagonal has twelve symmetry axis compared by eight symmetry axis for square, so, the hexagonal lattice is better suited for representing isotropic band limited signals leading to improving in packing density for images.

For higher symmetry degree, there are more isotropic filters can be designed and applied on hexagonal lattice [3]. The connectivity for the six neighbours of hexagonal cell made it the better for images edge detection [4, 5] and has been used for pattern recognition [6, 7]. Pattern recognition is the main parts in computer vision applications (CVA).

In various CVA widely used is the process of retrieving desired images from a large collection on the basis of features that can be automatically extracted from the images themselves. Image feature extraction is the process of generating features to be used in the classification task.

The most common visual features include colour, texture and shape, etc. [8-16], and most image annotation and retrieval systems have been constructed based on these features. In general, there are three methods for feature representation, which are global, block-based [17], and regionbased [18] features.

Clustering is a classical data analysis method that aims at grouping a set of objects into clusters. There are many methods used in image clustering and probabilistic methods $[19,20]$ are one of them. Evidential clustering algorithms (ECA) which based on probabilistic methods are using the theoretical framework of belief functions [21, 22]. Credal partition $\left(C_{p}\right)$ is a new concept of partition extends the existing concepts of hard, fuzzy and probabilistic partitions used in belief functions framework. For each region, $\left(C_{p}\right)$ consists of a mass of belief to any subset of the set of clusters. ECA is characterized by gain a deeper insight into the data and improve robustness with respect to outliers. Evidential C-Means (ECM) algorithm [22], which based on $\left(C_{p}\right)$ from data, is used in this paper. Image's feature classification have problem namely an uncertainty in labels of the training data.

To solve this problem, integration between two methods namely the belief functions and combining classifiers frameworks is suggested. The suggested approach which based on the supervised information is detecting labels inconsistencies of the learning data in each image's features space and assigning crisp and soft labels to them. Final decision about the class of a test pattern is then made by combining the belief functions produced by the base classifiers using Dempster's rule of combination.

The organization of this paper is described as: Hexagonal image processing in section 2. Section 3 describes the Hexagonal Region Feature Extraction Technique. Image's Feature Classification is introduced in section 4. Section 5 gives a brief description about Hexagonal Image retrieval. Section 6 is the experimental and results. Conclusion will be given section 7 .

\section{Hexagonal images processing:}

A computer vision system relies on three basic image processing components: acquisition, processing, and visualization. The generation of image data from a real source is handled by the acquisition stage which is equivalent to the human eye. Cameras, scanners, or more complex input devices are used for the acquisition stage. Image data processing involves manipulation to yield meaningful information by using a simple linear filtering algorithm or extracting descriptive details from image content structure that is considered more complicated methods. 
Integrating Hexagonal Image Processing with Evidential Probabilistic Supervised Classification Technique to Improve Image Retrieval Systems

While the visualization stage is essential for human observers as it is very useful for understanding the information generated by image processing. The integration between the computer vision system components results in a grid or a lattice from visual information, the lattice of interest is the hexagonal image processing (HIP) as shown in figure 1.

\section{Hexagonal Lattice Segmentation Technique:}

The hexagonal samples that were taken from the image can be called periodic monohedral tiling. Where monohedral tiling is the individual collection of closed sets that have the same size and shape which cover the Euclidean plane without gaps or overlap [23]. Hexagonal Lattice Segmentation (HLS) can be used to images segmented into regions if the pixels chromatography quantum within the region is significantly different from the surrounding regions. HLS is composed of two main factors are weight or the first moment of data, and orientation. Both factors are specified features extracted and analyzed from the attention window (AW). The basis of AW has sampled objects into the hexagonal array using the resampling scheme [24]. The data first moment is calculated as the sum of the individual pixel values by:

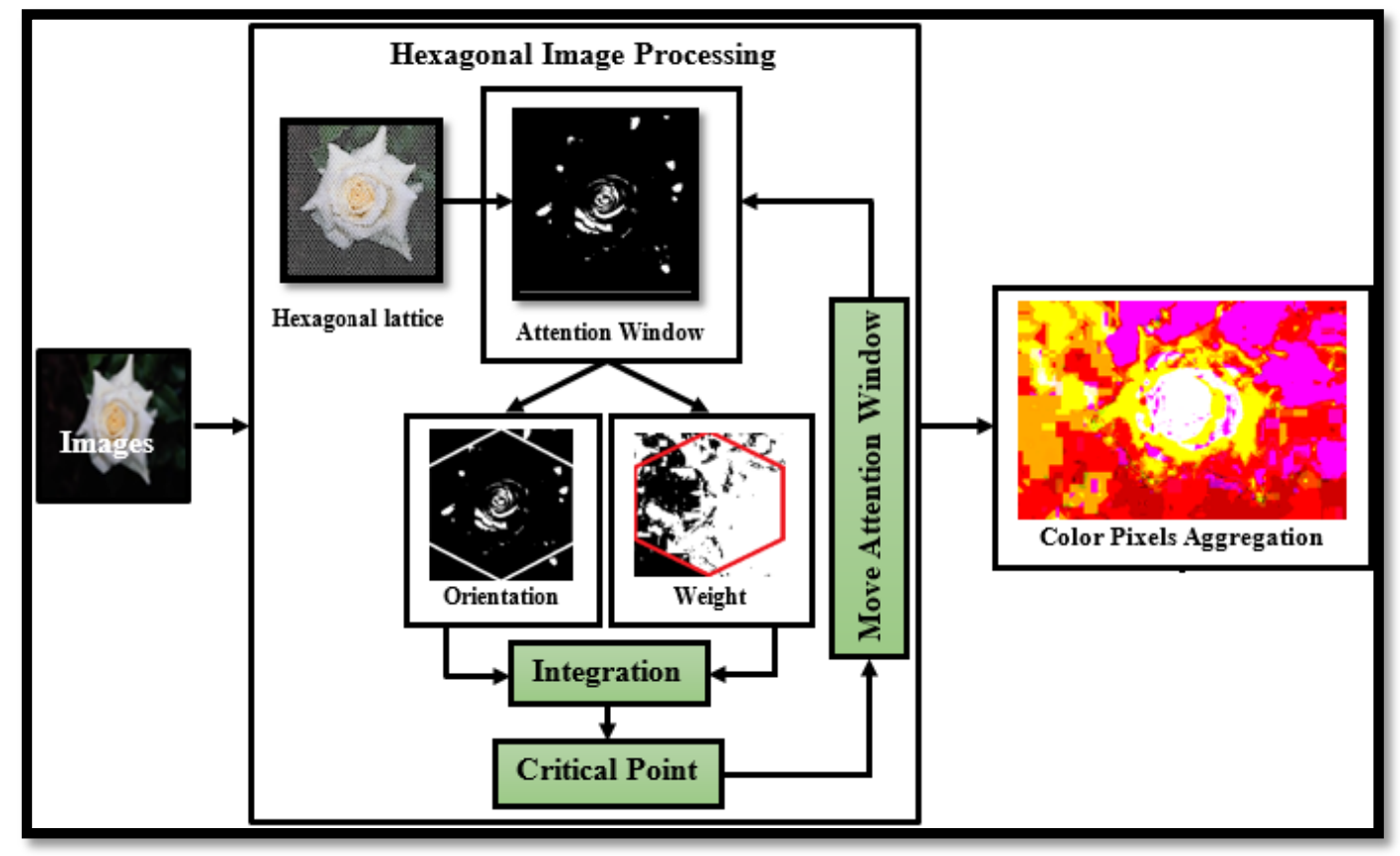

Fig. 1: Hexagonal Image Processing (HIP)

$$
w(x)=\sum_{i \in \mathbb{G}^{\lambda}} f(x \otimes i)
$$

Where, $f$ is the given $\lambda$-layer HIP.

Whenever the weight is low the accuracy of each region's boundaries is increased and the chance of overlap between the segmented regions is reduced. Normalization (i.e. dividing by the maximum value) is used to obtain normalized weights. This analysis is carried out in a hierarchical fashion [25] at all layers of the pyramid implicit within the HIP image.

There are several steps that are implemented for orientation calculation are Edge Detection, Skeletonization, and Orientation. Detection of edges from data is computed with relative efficiency by an edge detector. The skeletonization is performed to determine the edge map. Finally, the orientation phase takes place in which a series of 
orientation templates are matched to the data by analysing both the extracted weight and the routing of all layers of the pyramid.

Both of orientation and weight features which derived from each pyramid layers are arranged in rank order from most to least common. Then, the AW fixation point is determined by using a comparisons series between the candidate points. The highest vote in comparisons for most likely orientation is the next fixation point. A series of critical points which are candidate points for the next position of the AW is obtained. The current AW's center point has relative points which are stored as HIP indices. Based on commonality across resolution, the critical points are ranked with taking into account the lower resolution point with high frequent are tested first. To select a next movement to valid fixation point all the critical point is tested if does not involve visiting previously. Sometimes, a random movement is performed when effective candidate point is not found. The specific movement is stored as a HIP address and employed as an additive offset to the current attention window.

HLS technique is characterized by clustering of pixels that resulting from integration of hexagonal polygon units. Each cluster of pixels is shaped a region can be used to extract their own features. Then, the overall regions features are combined to form the image features.

\section{Feature Extraction:}

Features detection is generalized using a local power model [26]. There are types of filters that are orthogonal but same size spectra are used to filter the segmented hexagonal image: The local energy that calculated by the square root of the sum of the volume responses squared from these filters in each of the image regions as:

$$
E_{L}(I)=\sqrt{e^{2}(I)+o^{2}(I)}
$$

Where, $e(I)$ and $o(I)$ are the responses of an even-symmetric filter and an oddsymmetric filter, respectively. In the original, the edge or line feature corresponds to their maximal response. To implement this filter Gabor Functions (GF) is used. Gabor Functions (GF) are used to implement the filters, where characterized by their orientation and sensitivity can be tune easily.

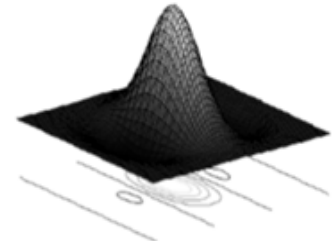

(a) even

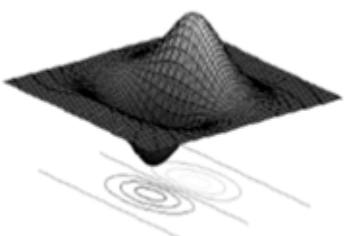

(b) odd

Fig. 2: Gabor function example

Figure 2 shows the symmetric even and odd Gabor functions, which consist of orthogonal phase and identical size spectra that can be selected. It is defined as follows:

$$
\begin{aligned}
& e(x, y)=e^{-\frac{\beta}{2}} \cos 2 \pi u_{0}(x \cos \alpha-y \sin \alpha) \\
& e(x, y)=e^{-\frac{\beta}{2}} \sin 2 \pi u_{0}(x \cos \alpha-y \sin \alpha) \\
& \beta=\left(\frac{x^{2}}{\sigma_{x}^{2}}+\frac{y^{2}}{\sigma_{y}^{2}}\right) \ldots \ldots \ldots \ldots \ldots \ldots \ldots \ldots \ldots \ldots \ldots \ldots \ldots \ldots \ldots \ldots \ldots \ldots \ldots
\end{aligned}
$$




\section{Integrating Hexagonal Image Processing with Evidential Probabilistic Supervised Classification Technique to Improve Image Retrieval Systems}

Where, $\alpha$ and $u_{0}$ are the orientation and the radial frequency (in cycles per image width) of the filter, respectively. Whereas, the filter falls in the $\mathrm{x}$ and $\mathrm{y}$ directions are $\sigma_{x}$ and $\sigma_{y}$, respectively. The following equations calculate the filter's frequency and orientation bandwidth values associated with $\sigma_{x}$ and $\sigma_{y}$ as follows:

$$
\begin{aligned}
\sigma_{x} & =\frac{\sqrt{2}}{2 \pi u_{0}} \frac{2^{B r}+1}{2^{B} r-1} \ldots \ldots \ldots \ldots \ldots \ldots \ldots \ldots \ldots \\
\sigma_{y} & =\frac{\sqrt{2}}{2 \pi u_{0} \tan \frac{B_{\theta}}{2}} \ldots \ldots \ldots \ldots \ldots \ldots \ldots
\end{aligned}
$$

The half-peak radial and frequency bandwidths are represented as $B_{r}$ (in octaves) and $B_{\theta}$ (in degrees) respectively as shown in figure 3. By varying the $\alpha$ in the Euclidean plane, we can determine different directions.

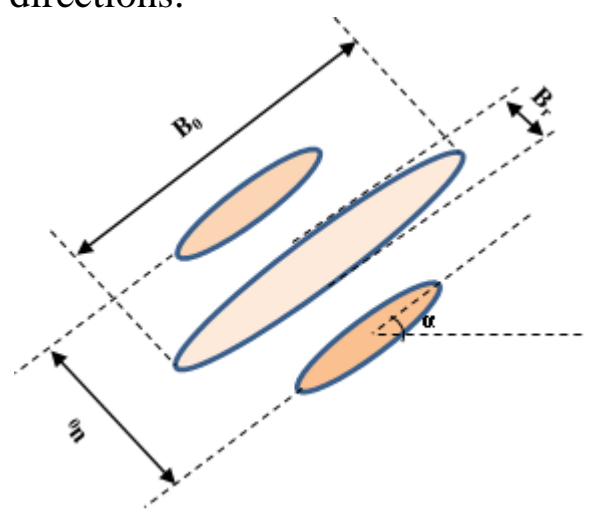

Fig. 3: Parameter to tune the Gabor filter

Calculation procedures can be speeded up by using frequency-domain filtering using HFFT. The original input image is converted to an approximately fixed-size HIP image regardless of the image's original size. Thus, the shape is extracted by selecting specific ranges of filter parameters for Gabor filters. 


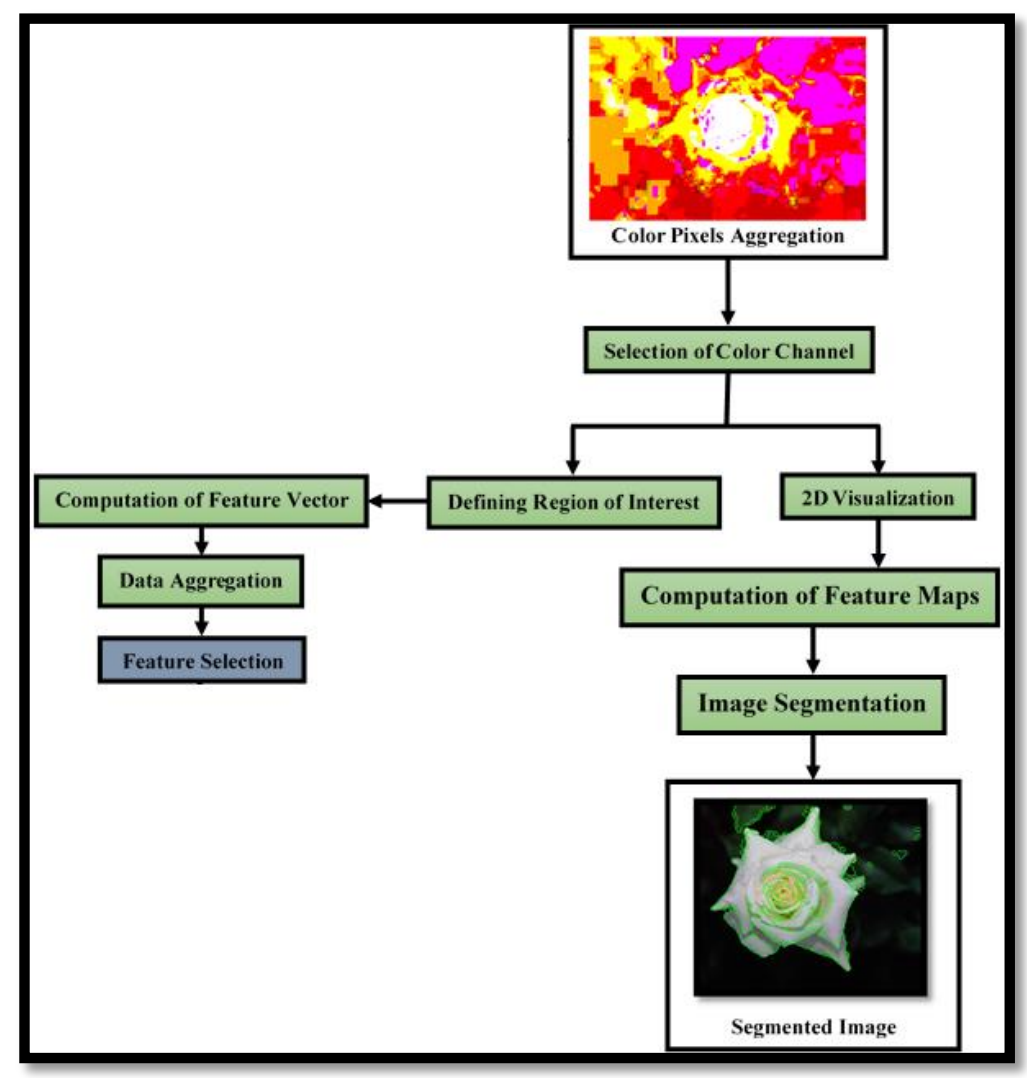

Fig. 4: Image Feature Extraction

\subsection{Feature Vectors:}

As shown in Figure 4, the local energy is calculated after filtering and thresholding, for each orientation and at each resolution. To normalize the resulting data, there is factor equal to the total energy (across all orientations) is used for a given resolution, so that the final feature vector is:

$$
C l_{R_{i j}}=\left[f_{R_{11}} \ldots f_{R_{1 \mathrm{i}}} \ldots f_{R_{21}} \ldots f_{R_{2 \mathrm{i}}} \ldots f_{R_{i j}}\right] \text {. }
$$

Where, the calculated features of orientation are $f_{R_{1 n}} \ldots f_{R_{n n}}$ at the lowest resolution and the $n^{\text {th }}$ (highest) resolution for computed orientation features are $f_{R_{n 1}} \ldots f_{R_{n n}}$. Hence, to calculate the individual feature values use:

$$
f_{R_{i j}}=\frac{e_{i j}}{\sum_{j=1}^{n} e_{i j}} .
$$

Where, the calculated energy is $e_{i j}$ in both channel $i^{\text {th }}$ resolution and $j^{\text {th }}$ orientation.

Feature extraction for each image $\left(F_{f_{R_{i j}}}^{i m_{k}}\right)$ is extracted and stored in database namely image features as:

$$
F_{f_{R_{i j}}}^{i m_{k}}=\left[\begin{array}{ccccccc}
f_{R_{11}}^{i m_{1}} & \ldots & f_{R_{1 n}}^{i m_{1}} & \ldots & f_{R_{n 1}}^{i m_{1}} & \ldots & f_{R_{n j}}^{i m_{1}} \\
\vdots & \vdots & \vdots & \ddots & \vdots & \vdots & \vdots \\
f_{R_{i 1}}^{i m_{k}} & \ldots & f_{R_{i n}}^{i m_{k}} & \ldots & f_{i n}^{i m_{k}} & \ldots & f_{i j}^{i m_{k}}
\end{array}\right]
$$




\section{Image's Feature Unsupervised Classification:}

\subsection{Evidence Theory:}

The theory of evidence allows the use of uncertain data [27]. Let $\Omega$ be a finite set of exclusive and exhaustive hypotheses, called the Discrimination Framework. The basic belief assignment (bba) is a function $\mathrm{m}$ from $2 \Omega$ to $[0,1]$ verifying:

$$
\left\{\begin{array}{c}
m(\varnothing)=0 \\
\sum_{A \subseteq \Omega} m(\varnothing)=1
\end{array}\right.
$$

For any $A \subseteq \Omega, m(A)$ represents the belief that one is willing to commit exactly to $A$, given a certain piece of evidence. The subsets $(A)$ from $\Omega(m(A)>0)$ are called the focal elements of $m$. Associated with $m$ are a belief or credibility function bel and a plausibility function $p l$, defined, respectively, for all $A \in \Omega$ as:

$$
\begin{aligned}
& \operatorname{bel}(A)=\sum_{B \subseteq A} m(B) \\
& \operatorname{pl}(A)=\sum_{A \cap B \neq \emptyset} m(B)
\end{aligned}
$$

The quantity bel(A) can be interpreted as a global measure of one's belief that hypothesis is true, while $\operatorname{pl}(\mathrm{A})$ may be viewed as the amount of belief that could potentially be placed in A, if further information became available [49]. The decision rule can be given by different approaches as following:

- Choose the maximum plausibility hypothesis $(p l)$;

- Choose the maximum pignistique probability hypothesis $(B e t P)$.

$$
\operatorname{BetP}(w)=\sum_{w \in A} \frac{m(A)}{|A|}
$$

\subsection{Evidence classification technique:}

Evidence classification is passing through two main steps; the first step image features clustering and classification are applied on the learning database separately. Whereas the second step consists to fuse the results of classification based on compromise between classifiers outputs. Both steps are contribution to solve the overlapping in clusters and bad learning in classification by using evidence theory model. The supervised evidence classification is used probabilistic model as mass function. Therefore, a mass function for each object $x$ belonging to a class $C l_{j}^{n}$, where $n$ is a number of classes. Each class $C l_{j}$ has:

$$
\begin{gathered}
m_{s}^{j}\left(C l_{j}\right)=\frac{\alpha_{s j} R_{s} p\left(C l_{j}^{f} \mid C l_{i}\right)}{1+R_{s} p\left(C l_{j}^{f} \mid C l_{i}\right)} \\
m_{s}^{j}\left(C l_{j}^{f}\right)=\frac{\alpha_{s j}}{1+R_{s} p\left(C l_{j}^{f} \mid C l_{i}\right)} \\
m_{s}^{j}\left(C l^{\text {unknown }}\right)=1-\alpha_{s j}
\end{gathered}
$$

Where, $C l_{j}^{f}$ : the class affected by the supervised classifier to the object $x$,

$C l_{j}$ : the real class

$$
\alpha_{s j} \text { : the reliability coefficient of the supervised classification for the class } C l_{j}^{f} \text {. }
$$

The conditional probabilities are estimated through the confusion matrix on the learning database: 


$$
\begin{gathered}
\alpha_{s j}=\max \left[p\left(C l_{j}^{f} \mid C l_{i}\right)\right] \quad \forall_{i}=\{1,2, \ldots, n\} \\
R_{s}=\max _{C l_{j}^{f}}\left[p\left(C l_{j}^{f} \mid C l_{i}\right)\right]^{-1} \ldots \ldots \ldots \ldots
\end{gathered}
$$

For the unsupervised evidence classification, mass functions must also be defined on the discernment space $\Omega$. However, the classes of $\Omega$ are unknown in clustering. Therefore the definition of mass function is made by measuring the similarities between clusters and classes found by the supervised classification. The similarity is calculated using recovery between clusters and classes. A class is considered similar to a cluster if it is recovered totally by the cluster. The proportions of found classes $C l_{1}^{f}, C l_{2}^{f}, \ldots, C l_{n}^{f}$ are looked for by the supervised classifier in each cluster [28]. The mass function for an object $x$ to be in the class $C l_{j}$ is as follows:

$$
m_{n s}\left(C l_{j}\right)=\frac{\left|C_{i} \cap C l_{j}^{f}\right|}{C_{i}}
$$

Where: $\left|C_{i}\right|:$ is the number of elements in the cluster $C_{i}$.

$$
\left|C_{i} \cap C l_{j}^{f}\right| \text { : is the number of elements in the intersection between } C_{i} \text { and } C l_{j}^{f} \text {. }
$$

Then we discount the mass functions as follows, $\forall A \in 2 \Omega$ by:

$$
\begin{array}{r}
m_{n s}^{\alpha_{i}}(A)=\alpha_{i} m_{n s}(A) \ldots \ldots \\
m_{n s}^{\alpha_{i}}(\Omega)=1-\alpha_{i}\left(1-m_{n s}(\Omega)\right)
\end{array}
$$

Where, $\alpha_{i}$ : is discounting coefficient depends on objects. An object situated in the center of cluster is considered more representative of the cluster than another one situated on the border i.e. the coefficient $\alpha_{i}$ is defined as $\left(v_{i}\right.$ is the center of cluster $\left.C_{i}\right)$ :

$$
\alpha_{i}=e^{-\left\|x-v_{i}\right\|^{2}}
$$

The mass functions for supervised and unsupervised classification are combined by using the conjunctive rule which take the maximum of pignistic probability as decision criterion.

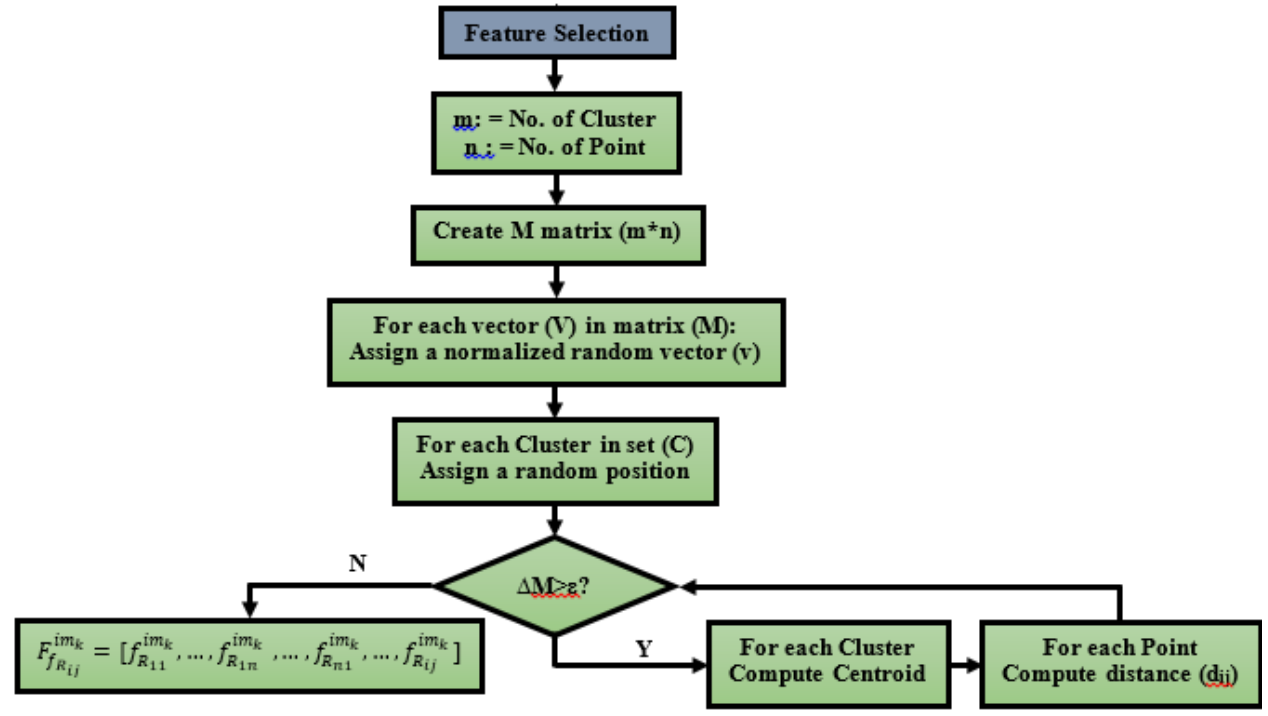

Fig. 5: Image Feature Classification. 


\subsection{Evidential C-means:}

In supervised classification step as shown in figure 5, Evidential C-means (ECM) is used which based on credal partition [29] using the theoretical framework of belief functions. The class membership of an image feature $F_{f_{R_{i j}}}^{i m_{k}}=\left[f_{R_{11}}^{i m_{k}}, \ldots, f_{R_{1 n}}^{i m_{k}}, \ldots, f_{R_{n 1}}^{i m_{k}}, \ldots, f_{R_{i j}}^{i m_{k}}\right]$ is represented by a basic belief assignment (bba) $m_{i}$ (.) over a given frame of discernment $\Omega=\left\{w_{1}, \ldots, w_{k}\right\}$. This representation is able to model all situations ranging from complete ignorance to full certainty concerning the class of $F_{f_{R_{i j}}}^{i m_{k}}$. In ECM, the mass of belief for associating the object $F_{f_{R_{i j}}}^{i m_{k}}$ with an element $A_{j}$ of $2 \Omega$ denoted by $m_{i j} \triangleq$ $m_{F_{f_{R_{i j}}}} m_{k}\left(A_{j}\right)$, is determined from the distance $d_{i j}$ between $F_{f_{R_{i j}}}^{i m_{k}}$ and the center vector $\bar{v}_{J}$ of the element $A_{j}$. The center vector $\bar{v}_{j}$ of $A_{j}$, is defined as the mean vector of the prototype attribute vectors of the singletons of $\Omega$ included in $A_{j} . \bar{v}_{j}$ is defined mathematically by:

$$
\bar{v}_{j}=\frac{1}{c_{j}} \sum_{i=1}^{c} s_{k j} v_{k}, \quad S_{k j}=\left\{\begin{array}{lr}
1, & \text { if } w_{k} \in A_{j} \\
0, & \text { Otherwise }
\end{array}\right.
$$

Where $v_{k}$ is the center attribute vector of class $w_{k}$, and $c_{j}=\left|A_{j}\right|$ denotes the cardinality of $A_{j}$, and $d_{i j}$ denotes the Euclidean distance between $F_{f_{R_{i j}}}^{i m_{k}}$ and $\bar{v}_{j}$. In ECM, the determination of $m_{i j} \triangleq m_{F_{f_{i j}}^{i m_{k}}}\left(A_{j}\right)$ is based on $d_{i j}$. Actually, $m_{i j}$ is obtained by the minimization of the following objective function $f_{E C M}^{\text {Reg }}$ under a constraint:

$$
f_{E C M}^{R e g}=\sum_{i=1}^{n} \sum_{\substack{A_{j} \subseteq \Omega \\ A_{j} \neq \varnothing}}\left(c_{j}^{\alpha} m_{i j}^{\beta} d_{i j}^{2}\right)+\sum_{i=1}^{n} \delta^{2} m_{i \emptyset}^{\beta}
$$

Subject to

$$
\sum_{A_{j} \subseteq \Omega}\left(m_{i j}+m_{i \emptyset}\right)=1
$$

The solution of the minimization of (eq. 4-15) under the constraint (eq. 4-16) has been established by Masson and Denœux in [30] and it is given for each object $F_{f_{i j}}^{i m_{k}},(\mathrm{k}=1,2$ ... m) by:

- For all $A_{j} \subseteq \Omega$ and $A_{j} \neq \varnothing$,

$$
m_{i j}=\frac{c_{j}^{-\alpha /(\beta-1)} d_{i j}^{-2 /(\beta-1)}}{\sum_{A_{k} \neq \varnothing}\left[c_{k}^{-\alpha /(\beta-1)} d_{i k}^{-2 /(\beta-1)}+\delta^{-2 /(\beta-1)}\right]}
$$

Where $\alpha$ is a tuning parameter allowing to control the degree of penalization; $\beta$ is a weighting exponent (its suggested default value in [9] is $\beta=2$ ); $\delta$ is a given threshold tuning parameter for the filtering of the outliers; $c_{j}=\left|A_{j}\right|$ is the cardinality of the set $A_{j}$.

- For $A_{j} \neq \emptyset$,

$$
m_{i \emptyset} \triangleq m_{F_{f_{R_{i j}}}^{i m_{k}}}(\varnothing)=1-\sum_{A_{j} \neq \varnothing} m_{i j}
$$

The centers of the class are given by the rows of the matrix $V_{c \times p}$.

$$
V_{c \times p}=H_{c \times c}^{-1} \cdot B_{c \times p}
$$


Where the elements $B_{l q}$ of $B_{c \times p}$ matrix for $l=1,2, \ldots, c, q=1,2, \ldots p$, and the elements $H_{l k}$ of $H_{c \times c}$ matrix for $l, k=1,2, \ldots, c$ are given by:

$$
\begin{gathered}
B_{l q}=\sum_{i=1}^{n}\left(F_{f_{R_{i j}}}^{i m_{k}}\right)_{q} \sum_{w_{l} \in A_{j}} c_{j}^{\alpha-1} m_{i j}^{\beta} \\
H_{l k}=\sum_{i=1}^{n} \sum_{\left\{w_{k}, w_{l}\right\} \subseteq A_{j}} c_{j}^{\alpha-2} m_{i j}^{\beta}
\end{gathered}
$$

\section{Hexagonal Image retrieval:}

From a technical standpoint; the image retrieval systems are based on sequential functions defined as querying, extract features, matching measures, indexing data structures, relevance feedback, then display the result as shown in figure 6 .

The image is segmented into at regions by using clustering of pixel color in image hexagonal lattice. The extracted features of image regions are aggregated to form the image features. Features of images are classified into categories and stored in a database. The query image also is specified by using the same method and extracted its feature. The system can indicate the relative importance of color and shape.

In the matching first, a correspondence between regions in the query feature and database of images features have detected the category which the image belongs to it. The similarity between query and images is divided into two types of similarities. Firstly, regions similarity which belong to template matching and based on the number of pixel. Secondly, the color similarity which based on chromatography quantum for pixel and the distance in HLS space between the uniform region colors.

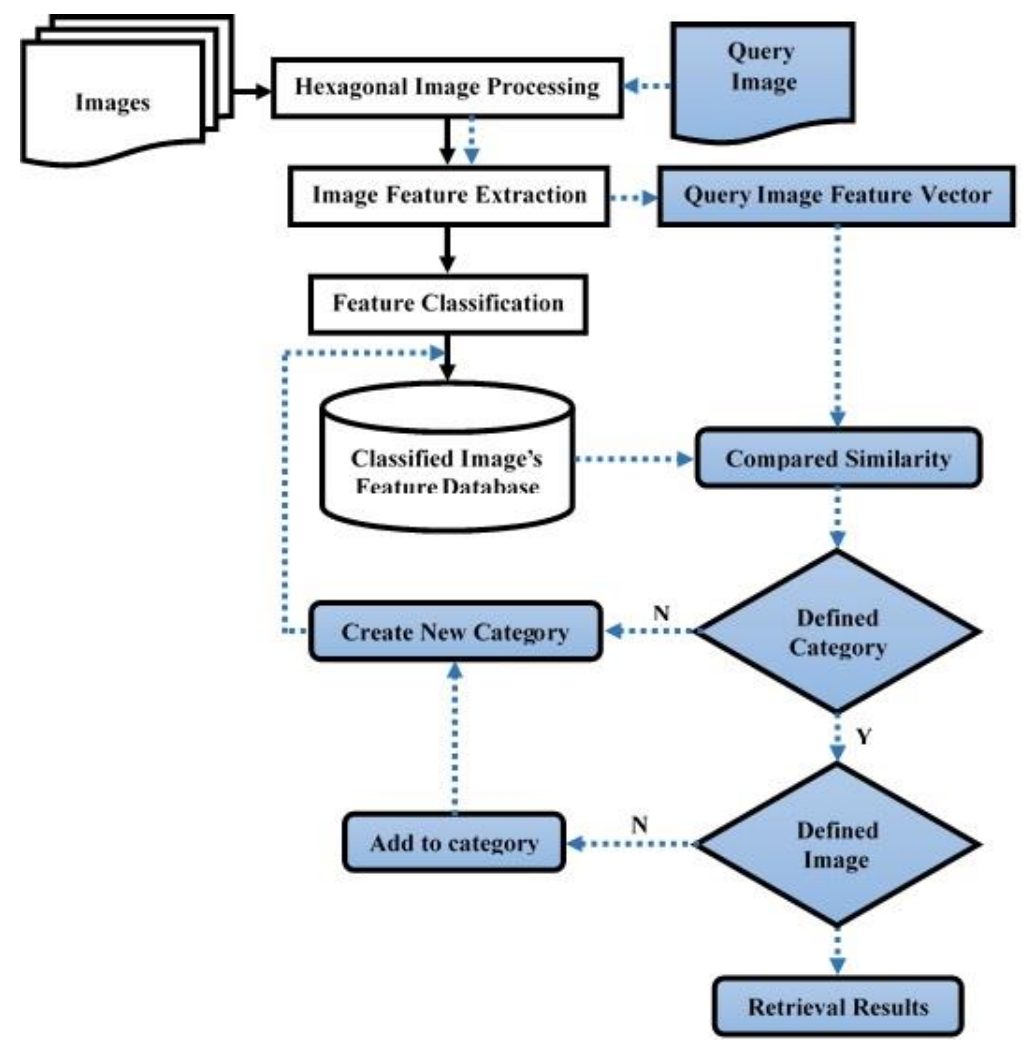

Fig. 6: Hexagonal image retrieval system. 
Integrating Hexagonal Image Processing with Evidential Probabilistic Supervised Classification Technique to Improve Image Retrieval Systems

In indexing step, the image feature's is divided into region's features (RF). Each RF is converted into a hash index by the multi-hash method [31]. The index points to the appropriate region containing pointers to one or more RF classes.

\section{Experimental and Results:}

The Wavelet-Based Image Indexing and Searching (WBIIS) database [32] is used to evaluate the proposed system (HIRS). WBIIS image database consists of 10,000 generic images with variety size distributed on 10 categories of Africa, Beaches, Building, Buses, Dinosaurs, Elephants, Flowers, Foods, Horses and Natural. Several test images were used during experimentation and result as shown in figure 7.

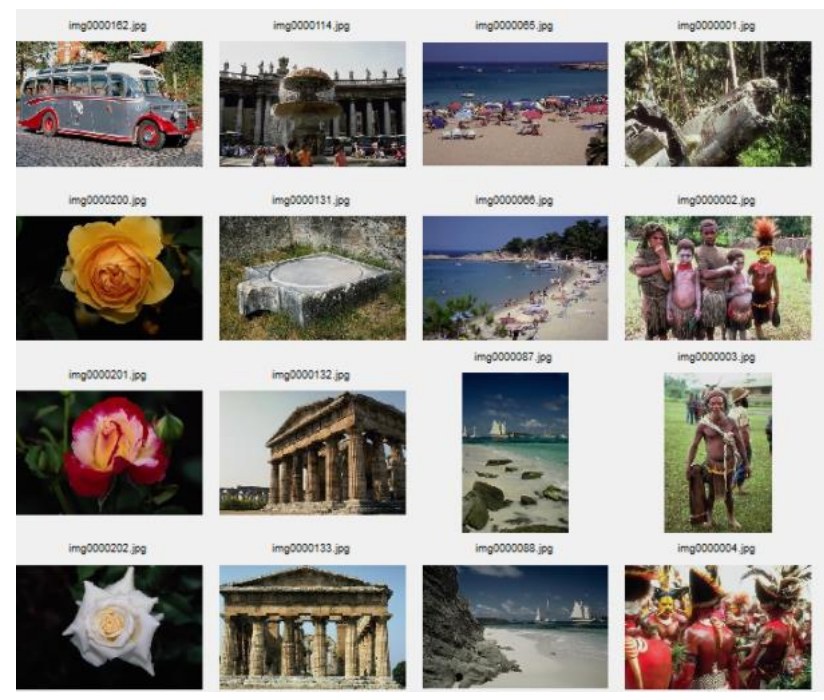

Fig. 7: Database categories

Image enhancement is the processing of images to increase their usefulness. There are several objectives are accomplished to image enhanced such as Hexagonal Lattice image, Hexagonal Image processing, Object region detection, and Aggregate quantum chromatography for pixels in each region as shown in figure 8.

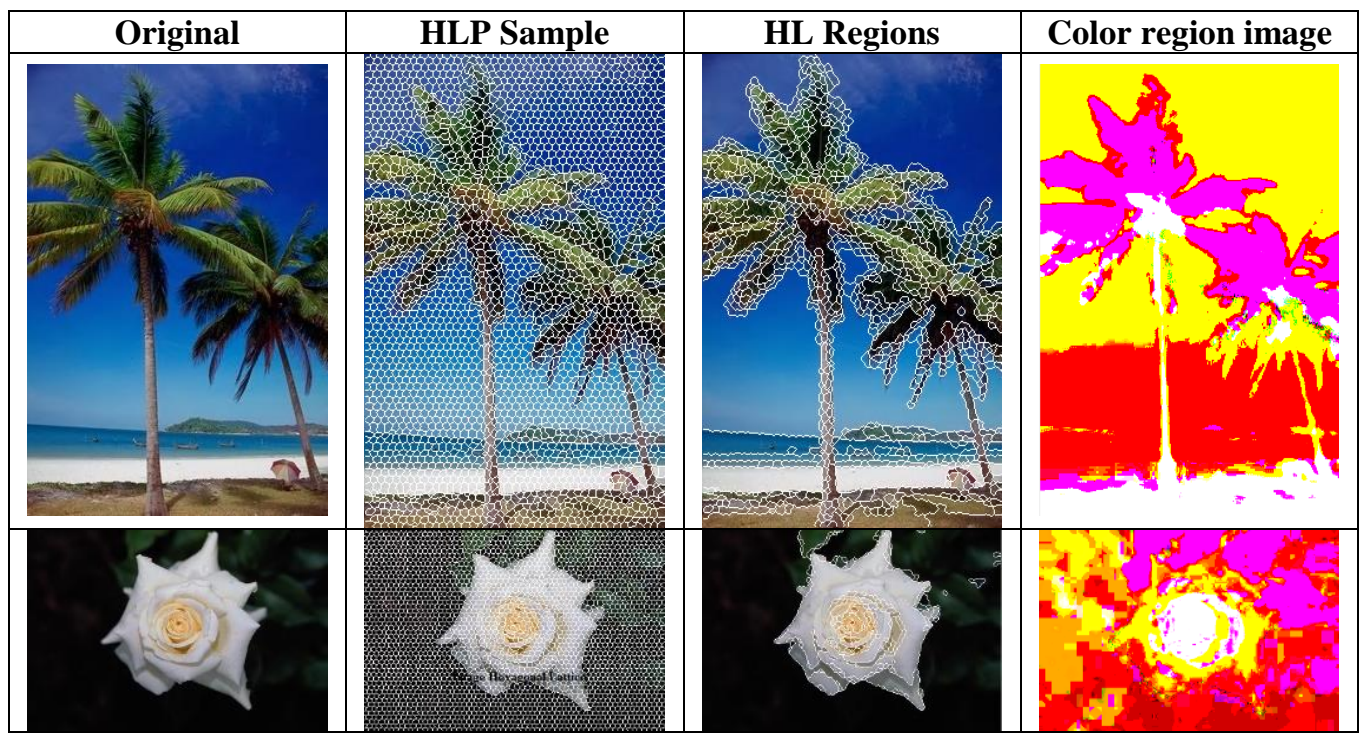




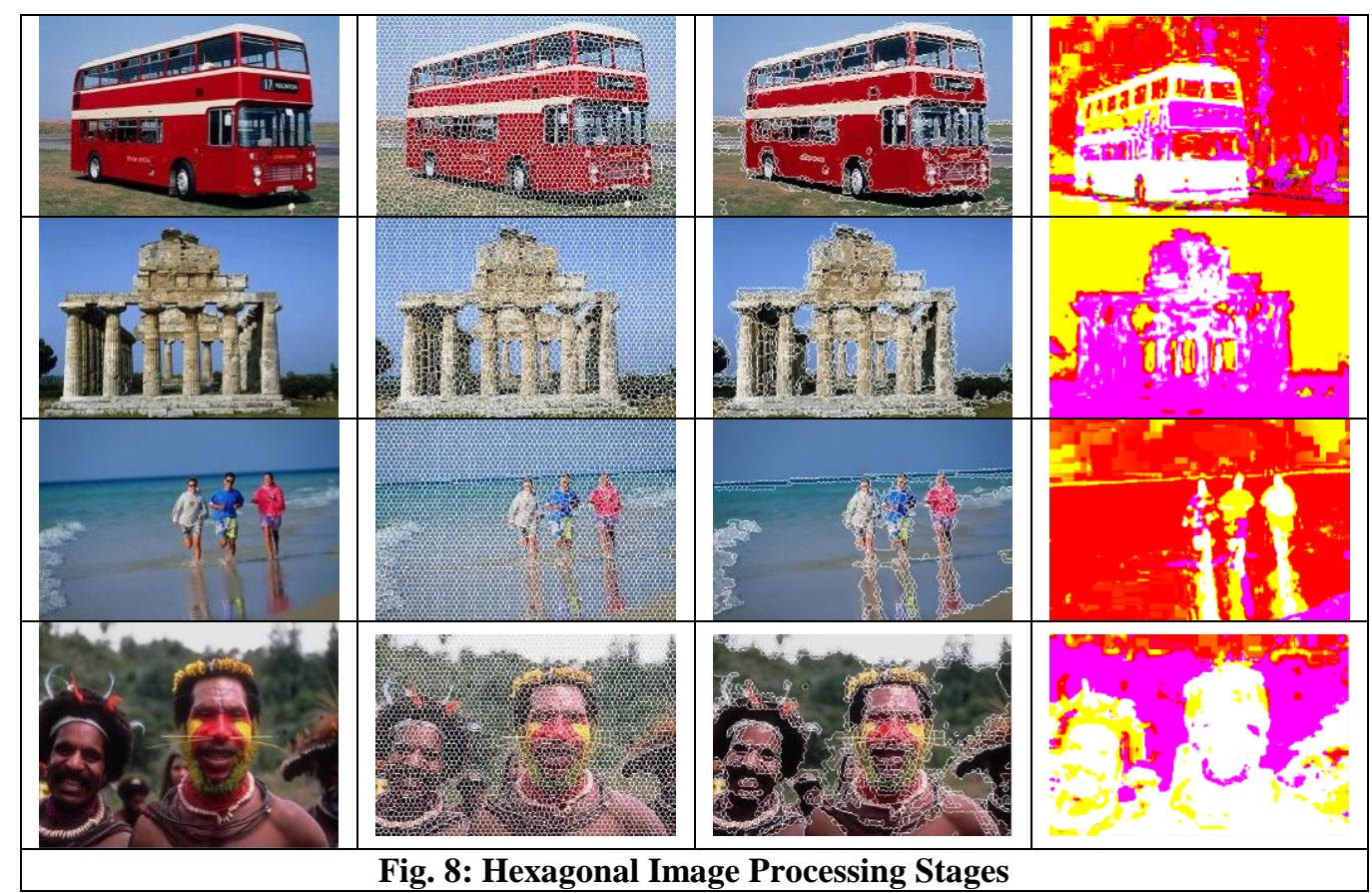

Gabor Wavelet filter $(\mathrm{GW})$ is used to extract features for each region. The features are found by calculating the mean and variance of the $\mathrm{GW}$ image regions. A feature vector is generated for an image feature, for example, if a $5 \times 8 \mathrm{GW}$ set is used, then there will be 80 in this feature vector. The element order in their method is based on the orientation, where the first 8 elements are ordered as $0^{\circ}, 22.5^{\circ}, 45^{\circ}, 67.5^{\circ}, 90^{\circ}, 112.5^{\circ}, 135^{\circ}, 157.5^{\circ}$ of the same scale with $22.5^{\circ}$ detected as the dominant direction as shown in figure $9(\mathrm{a}-\mathrm{b})$. For each region, the features are normalized by the maximum value in each set of feature vector. All regions features are aggregate to represents the image feature vector. For example, one image and its corresponding Gabor wavelet features (Mean and Variance) are shown in figure 10.

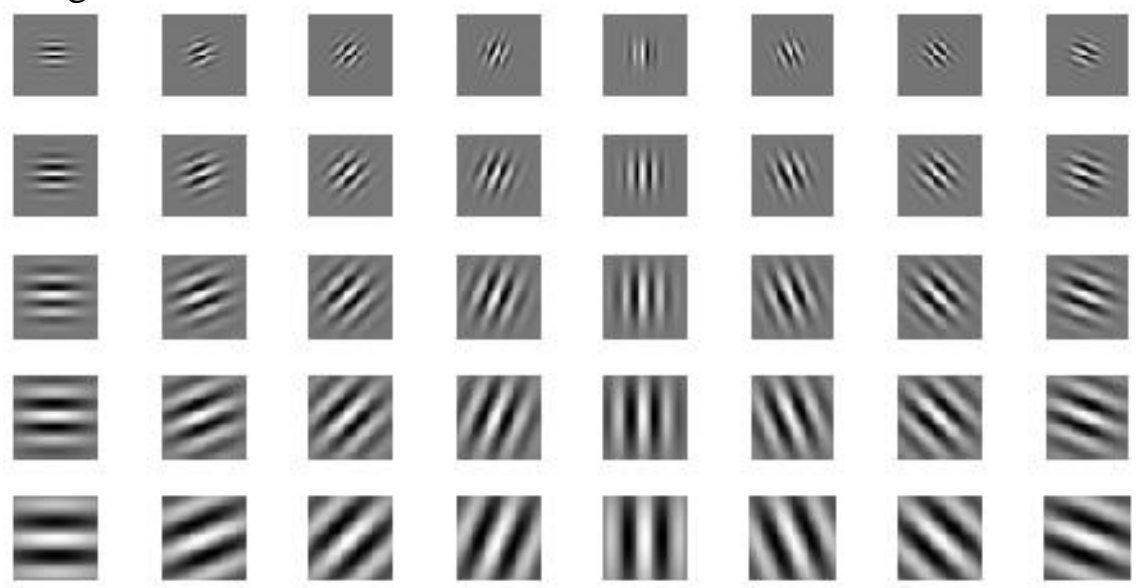

Fig.9-a: Gabor wavelet representation as real parts of Gabor filter 
Integrating Hexagonal Image Processing with Evidential Probabilistic Supervised Classification Technique to Improve Image Retrieval Systems

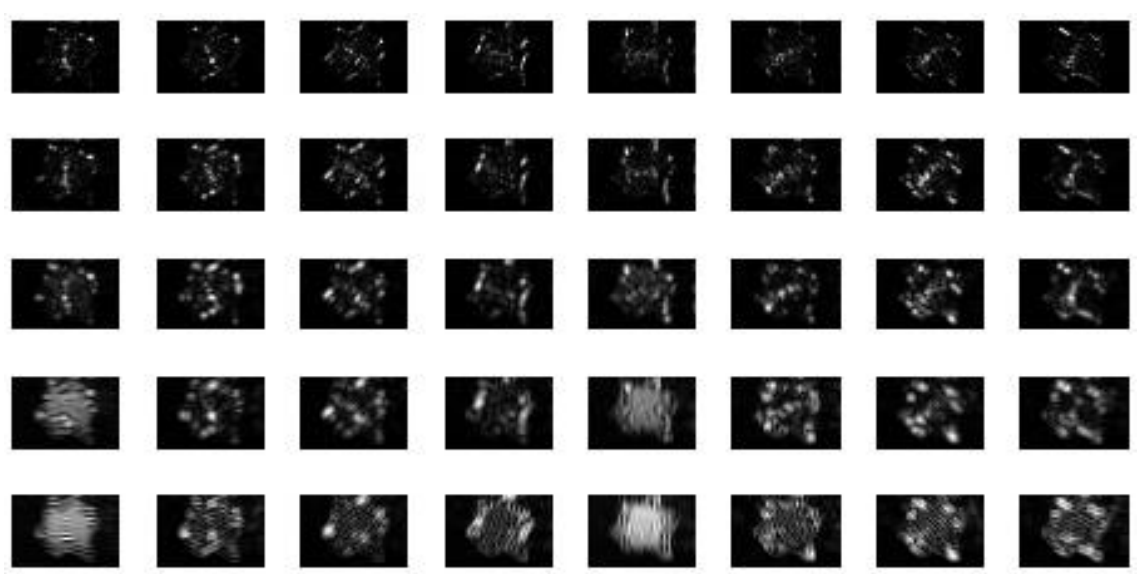

Fig. 9-b: Gabor wavelet representation as magnitudes of Gabor filter

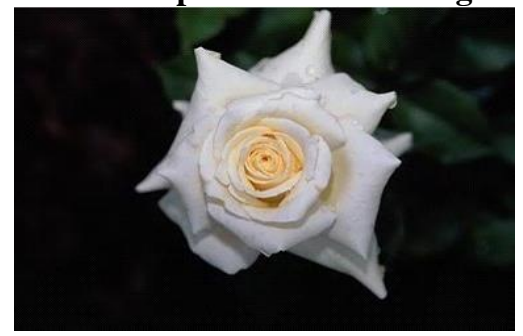

(a) The 40 mean magnitude features

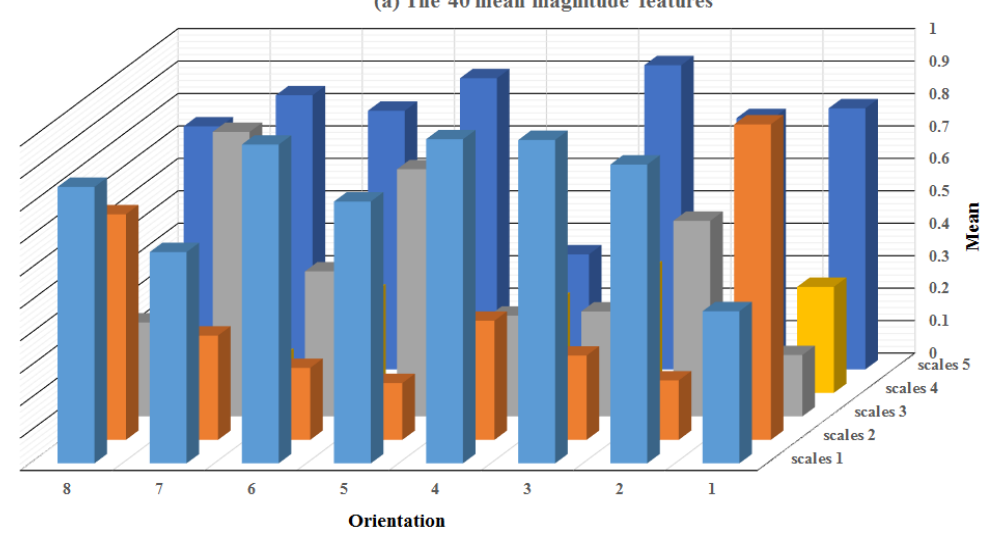

(b) The 40 varience features

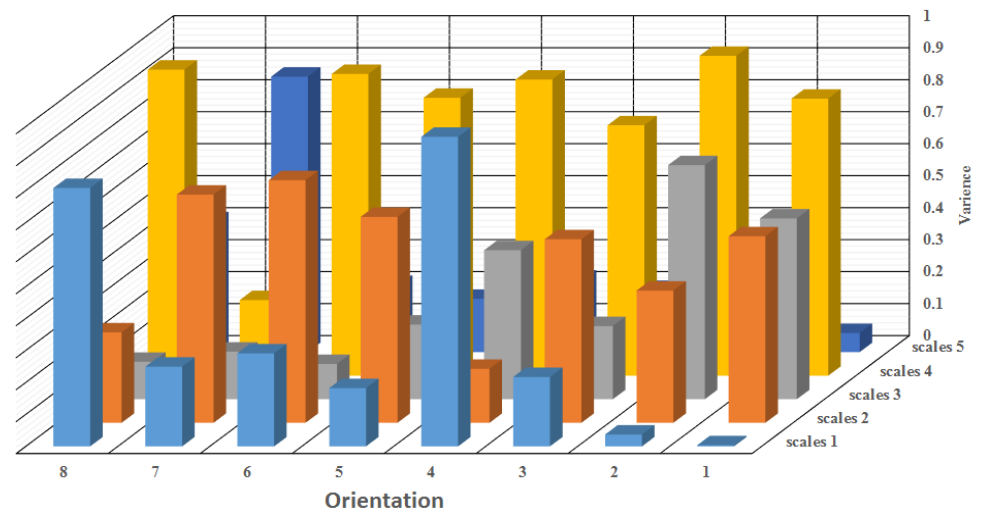

Fig. 10: The features extracted from three images based on a $5 \times 8$ Gabor wavelet set.

Evidential C-means (EC-Means) is used to clustering the image's features by determine the cluster belief as a distances between the object and corresponding centers of cluster's. That is solving the overlapping problem between clusters which represents in overlap of 
different cluster's centers or is very close. Image's features are clustered into five categories and represents image feature attributes as shown in figure 11.

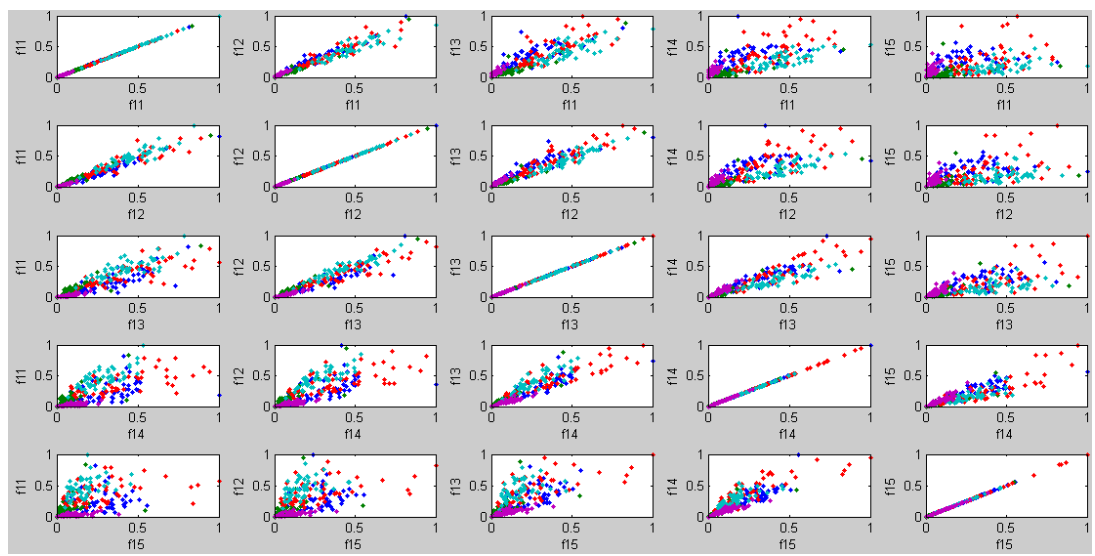

Fig. 11: Attribute image's features clusters

In each step of the iteration, the cluster centers and the belief function are updated and the objective function is minimized to find the best location for the clusters. The process stops when the maximum number of iterations is reached, or when the objective function improvement between two consecutive iterations is less than the minimum amount of improvement specified as shown in figure 12.

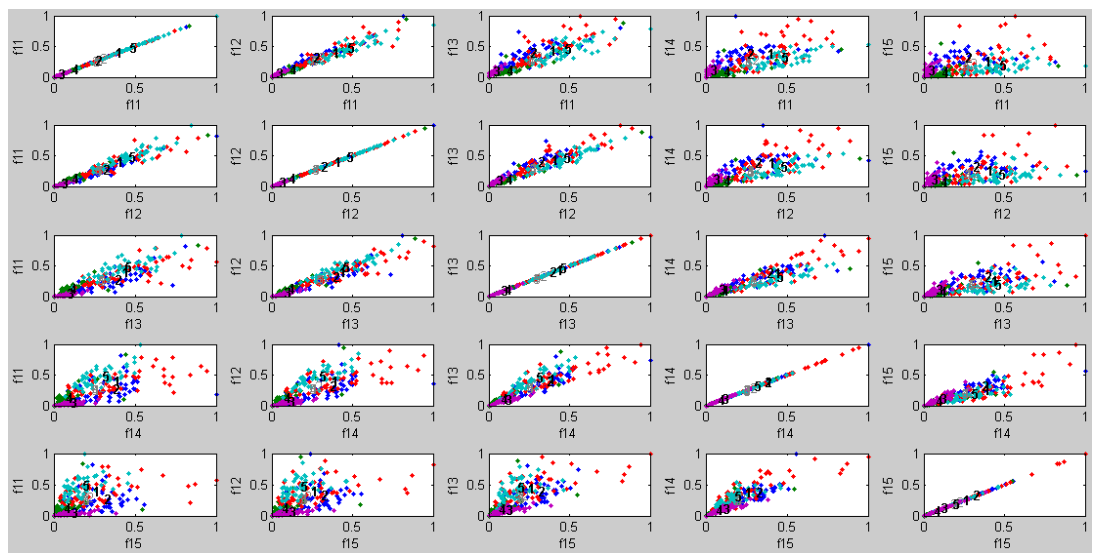

Fig. 12: Images clustering by EC-Mean.

For each experiment, one image is randomly selected as a query image from the database and then obtained the retrieved images as shown in figure 13.
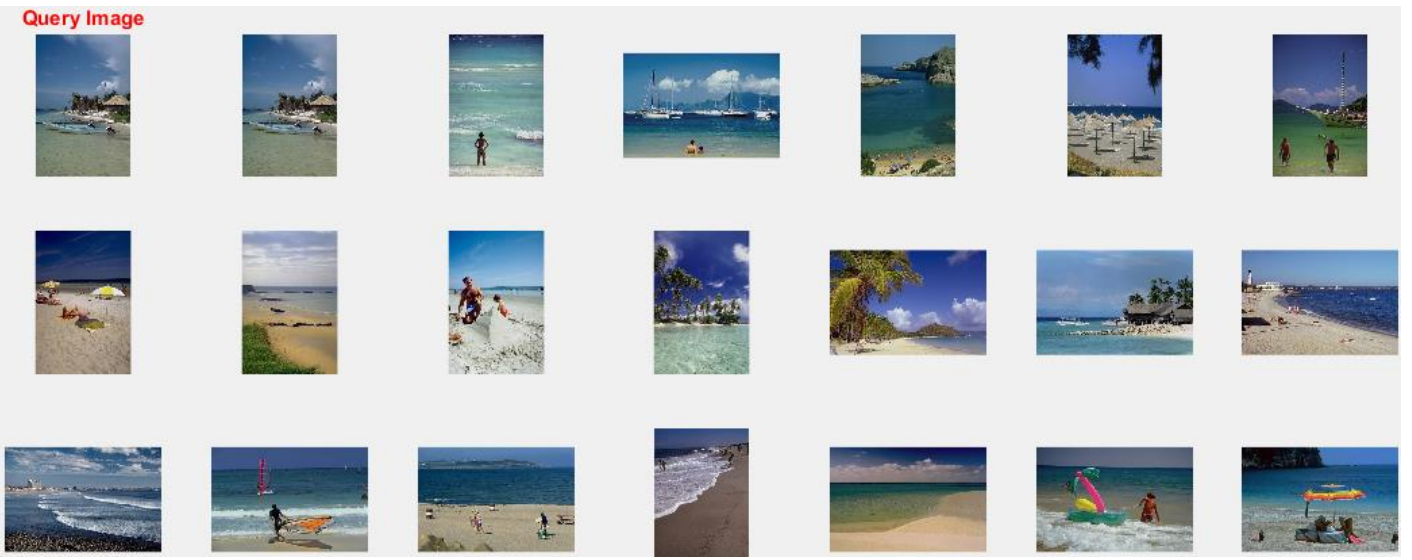
Fig. 13: example for image retrieval

\subsection{Performance Measures:}

To provide numerical results, 30 sample images chosen randomly from 10 categories for test, each containing three of the images. The most common evaluation measures used in image retrieval system are Precision and Recall are defined as:

$$
\begin{gathered}
\text { Precision }(P)=\frac{\text { No. relevant images retrieved }}{\text { Total No.images retrieved }} \ldots . \\
\text { Recall }(R)=\frac{\text { No. relevant images retrieved }}{\text { Total No.images retrieved in the catigory }}
\end{gathered}
$$

Precision versus Recall graph (PR graph) is usually used to show the relation between (P) and $(\mathrm{R})$ as shown in figure 14. Since PR graphs may not contain all the desired information, so there are several measures based on precision and recall are used [33].
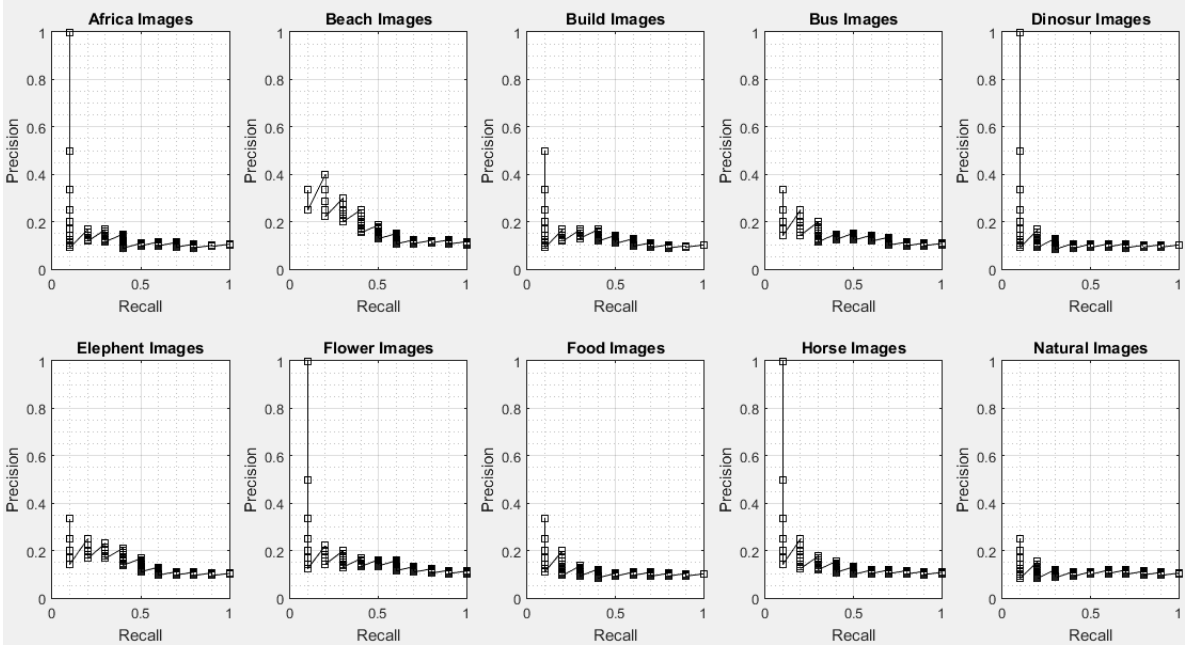

Fig. 14: P-R graph for three image query for each categories in WBIIS database

One of these measures is defined as the average rank (AR) which depends on both the collection size $(\mathrm{N})$ and the number of relevant images $\left(N_{R}\right)$ for a given query. Consequently, AR is normalized by these numbers and propose the normalized average $\operatorname{rank}(\bar{R})$ :

$$
\bar{R}=\frac{1}{N N_{R}}\left(\sum_{i=1}^{N_{R}} R_{i}-\left(\frac{N_{R}\left(N_{R}-1\right)}{2}\right)\right)
$$

Where $R_{i}$ is the rank at which the $i^{\text {th }}$ relevant image is retrieved. This measure is 0 for perfect performance, and approaches 1 as performance worsens. For random retrieval the result would be $0.5 . R_{1}$ is a simpler and more robust measure is the rank of the first relevant image, which is used in WBIIS and it is very useful for content-based image retrieval as well. Table. 1 is shown of $\overline{\boldsymbol{R}}$ value for Hexagonal image retrieval system of the same query that used in figure 14, and compared with image retrieval system based on square lattice. Comparison between AR results of proposed system and image retrieval system based on square lattice shown that:

HIRS is better than another system where R' values close to zero that mean the proposed system performance is approaching the perfect. While the most of R' values for other system is close to 0.5 i.e. approaching to randomly performance. 
Table 1: Performance measures for 3 different query for each category. Query Hexagonal image retrieval system Image retrieval based on square lattice

\begin{tabular}{|c|c|c|c|c|c|c|c|c|}
\hline & N & NR & $\boldsymbol{R}_{\mathbf{1}}$ & $\overline{\boldsymbol{R}}$ & $\mathrm{N}$ & $\mathrm{NR}$ & $\boldsymbol{R}_{\mathbf{1}}$ & $\overline{\boldsymbol{R}}$ \\
\hline Africa & 1000 & 40 & 3 & 0.0033 & 1000 & 25 & 2 & 0.22341 \\
\hline Beach & 1000 & 35 & 15 & 0.007714 & 1000 & 42 & 23 & 0.0357 \\
\hline Build & 1000 & 31 & 1 & 0.00329 & 1000 & 35 & 11 & 0.1206 \\
\hline Bus & 1000 & 26 & 4 & 0.005038 & 1000 & 45 & 24 & 0.0923 \\
\hline Dinosaur & 1000 & 35 & 8 & 0.000229 & 1000 & 15 & 16 & 0.345 \\
\hline Elephant & 1000 & 20 & 12 & 0.0211 & 1000 & 12 & 5 & 0.2415 \\
\hline Flower & 1000 & 39 & 7 & 0.001641 & 1000 & 45 & 102 & 0.328 \\
\hline Food & 1000 & 21 & 3 & 0.029714 & 1000 & 45 & 62 & 0.593 \\
\hline Horse & 1000 & 34 & 1 & 0.000618 & 1000 & 23 & 7 & 0.314 \\
\hline Natural & 1000 & 26 & 1 & 0.012038 & 1000 & 52 & 12 & 0.0526 \\
\hline
\end{tabular}

\section{Conclusion:}

In this paper, both of hexagonal image processing and evidential theory are used to obtain high accuracy in images classification. The hierarchical nature of the hexagonal image processing addressing scheme is exploited to extract features from the image efficiently. Hexagonal image processing performed well in images contours detection and high capturing of image content regions. The aims of hexagonal image processing is implemented by aggregated the interest neighboring pixels together and follows contours to find the shape where it has a high ability to change direction and curvature. Evidential theory is used in both of supervised and unsupervised learning for image classification. In unsupervised learning, A C-means clustering method based on the framework of belief functions has used to overcome on overlapped image clusters.in belief C-means method, both the distance between the image and the images cluster's center is token in account and also the distances between the image and the centers of the involved specific image clusters in order to compute the belief of the imprecise images cluster's. Whereas in supervised learning, Dempster-Shafer evidential reasoning and Artificial Neural Network (ANN) classifiers are used to images database classification as alternative techniques to maximum likelihood. The results are shown that evidential reasoning and neural network approaches is suited for high accuracy images classification.

\section{Reference:}

1. D. P. Petersen and D. Middleton, "Sampling and Reconstruction of Wave Number-Limited Functions in N-Dimensional Euclidean Spaces," Information and Control, vol. 5, pp. 279323, 1962

2. R. M. Mersereau, The processing of hexagonally sampled two-dimensional signals. Proc. IEEE, vol. 67, pp. 930-949, June 1979.

3. W. Li and A. Fettweis, Interpolation filters for 2-D hexagonally sampled signals, Int. J. Circuit Theory Applic., vol. 25, pp. 259-277, 1997.

4. R. C. Staunton, "The design of hexagonal sampling structures for image digitization and their use with local operators," Image and Vision Computing, vol. 7, no. 3, pp. 162-166, 1989 . 
5. L. Middleton and J. Sivaswamy, Edge detection in a hexagonal-image processing framework, Image and Vision Computing, vol. 19, no. 14, pp. 1071-1081, Dec. 2001.

6. A. Laine, and S. Schuler, Mammographic feature enhancement by multiscale analysis, IEEE Trans. Med. Imag. vol. 13, pp. 725-740, Dec. 1994.

7. R. C. Staunton, “One-pass parallel hexagonal thinning algorithm,” in Proc. Inst. Elect. Eng. Vision, Image and Signal Processing, vol. 148, 2001, pp. 45-53.

8. T. K. Shih, J. Y. Huang, C. S. Wang, et al., "An intelligent content-based image retrieval system based on color, shape and spatial relations", In Proc. National Science Council, R. O.C., Part A: Physical Science and Engineering, vol. 25, no. 4, (2001), pp. 232-243.

9. P.L. Stanchev, D. Green, Jr. and B. Dimitrov, High level colour similarity retrieval, International Journal of Information Theories and Applications, vol. 10, no. 3, (2003), pp. 363-369.

10. N. C. Yang, W. H. Chang, C. M. Kuo, et al., "A fast MPEG-7 dominant colour extraction with new similarity measure for image retrieval", Journal of Visual Comm. and Image Retrieval, vol. 19, (2008), pp. 92-105.

11. M. M. Islam, D. Zhang and G. Lu, A geometric method to compute directionality features for texture images, In Proc. ICME, (2008), pp. 1521-1524.

12. S. Arivazhagan and L. Ganesan, "Texture classification using wavelet transform", Pattern Recognition Letters, vol. 24, (2003), pp. 1513-1521.

13. S. Li and S. Shawe-Taylor, "Comparison and fusion of multi-resolution features for texture classification", Pattern Recognition Letters, vol. 26, no. 5, (2005), pp. 633-638.

14. W. H. Leung and T. Chen, "Trademark retrieval using contour-skeleton stroke classification", In Proc. ICME, (2002), pp. 517-520.

15. Y. Liu, J. Zhang, D. Tjondronegoro, et al., A shape ontology framework for bird classification, In Proc. DICTA, (2007), pp. 478-484.

16. C. F. Tsai, "Image mining by spectral features: A case study of scenery image classification", Expert Systems with Applications, vol. 32, no. 1, (2007), pp. 135-142.

17. H. Lu, Y. B. Zheng, X. Xue, et al., Content and context-based multi-label image annotation, In Proc. Workshop of CVPR, (2009), pp. 61-68.

18. T. W. S. Chow and M. K. M. Rahman, "A new image classification technique using treestructured regional features", Neurocomputing, vol. 70, no. 4-6, (2007), pp. 1040-1050.

19. R. Krishnapuram and J. Keller, "A possibilistic approach to clustering," IEEE Transactions on Fuzzy Systems, vol. 1, no. 2, pp. 98-110, 1993.

20. S. Sen and R. Dave', "Clustering of relational data containing noise and outliers," in Fuzzy Systems Proceedings, vol. 2, 1998, pp. 98-110.

21. T. Denœux and M. Masson, "EVCLUS: evidential clustering of proximity data," IEEE Trans. Systems, Man and Cybernetics: B, vol. 34, pp. 95-109, 2004.

22. M.-H. Masson and T. Denœux, "ECM: An evidential version of the fuzzy c-means algorithm," Pattern Recognition, vol. 41, pp. 1384-1397, 2008.

23. B. Gr"unbaum and G. Shephard, "Tilings and Patterns", W. H. Freeman and Company (New York), 1987.

24. Middleton, Lee, and Jayanthi Sivaswamy. Hexagonal image processing: A practical approach. Springer Science \& Business Media, 2006.

25. Montanvert, Annick, Peter Meer, and Azriel Rosenfeld. "Hierarchical image analysis using irregular tessellations." IEEE transactions on pattern analysis and machine intelligence 13.4 (1991): 307-316. 
26. M. Morrone and D. Burr, "Feature Detection in Human Vision: A phase dependent energy model," Proceedings of the Royal Society, vol. B235, pp. 221-245, 1988.

27. Shafer, Glenn. A mathematical theory of evidence. Vol. 1. Princeton: Princeton university press, 1976.

28. De'ath, Glenn, and Katharina E. Fabricius. "Classification and regression trees: a powerful yet simple technique for ecological data analysis." Ecology 81.11 (2000): 3178-3192.

29. Masson, Marie-Hélène, and Thierry Denoeux. "ECM: An evidential version of the fuzzy cmeans algorithm." Pattern Recognition 41.4 (2008): 1384-1397.

30. Denœux, Thierry, and M-H. Masson. "EVCLUS: evidential clustering of proximity data." Systems, Man, and Cybernetics, Part B: Cybernetics, IEEE Transactions on 34.1 (2004): 95109.

31. Wang, Jingdong, et al. "Hashing for similarity search: A survey." arXiv preprint arXiv: 1408. 2927 (2014).

32. Jia Li and James Z. Wang, "Real-time Computerized Annotation of Pictures," IEEE Transactions on Pattern Analysis and Machine Intelligence, vol. 30, no. 6, pp. 985-1002, 2008.

33. Geiger, Andreas, Philip Lenz, and Raquel Urtasun. "Are we ready for autonomous driving? the kitti vision benchmark suite." Computer Vision and Pattern Recognition (CVPR), 2012 IEEE Conference on. IEEE, 2012. 